\title{
The role of attentional breadth in perceptual change detection
}

\author{
HEATHER L. PRINGLE, DAVID E. IRWIN, and ARTHUR F. KRAMER \\ University of Illinois at Urbana-Champaign, Urbana, Illinois \\ and \\ PAUL ATCHLEY \\ University of Kansas, Lawrence, Kansas
}

\begin{abstract}
Previous research has shown that changes to scenes are often surprisingly hard to detect. The research reported here investigated the relationship between individual differences in attention and change detection. We did this by assessing participants' breadth of attention in a functional field of view task (FFOV) and relating this measure to the speed with which individuals detected changes in scenes. We also examined how the salience, meaningfulness, and eccentricity of the scene changes affected perceptual change performance. In order to broaden the range of individual differences in attentional breadth, both young and old adults participated in the study. A strong negative relationship was obtained between attentional breadth and the latency with which perceptual changes were detected; observers with broader attentional windows detected changes faster. Salience and eccentricity had large effects on change detection, but meaning aided the performance of young adults only and only when changes also had low salience.
\end{abstract}

Our representation of the visual details of a scene affects our ability to detect changes to that scene. Findings for a limited transsaccadic representation of details (e.g., Irwin, 1996) imply that the ability to report changes in scenes will also be limited. Indeed, recent research shows that when changes to scenes coincide with saccadic eye movements, we have a limited ability to detect them (e.g., Grimes, 1996; Henderson, 1997; McConkie \& Currie, 1996). Even studies simulating saccadic eye movements suggest that large and conspicuous scene changes can take a substantial amount of time to detect (Rensink, O'Regan, \& Clark, 1997).

On the basis of these findings, researchers have hypothesized that the perception of a change in a scene occurs when attention is directed to the part of the scene being changed. However, the link between attention and change detection has only been suggested by the research, and not yet convincingly established. For example, Rensink et al. (1997) investigated observer latencies in detecting changes to 48 photographs of everyday scenes. A change was made to a single object in each scene and was scored independently as having central or marginal in-

This research was supported by a grant from the National Institute on Aging (AG14966) and a cooperative research agreement with the Army Research Laboratory (DAAL01-96-0003). We thank Ron Rensink and an anonymous reviewer for helpful comments on an earlier version of the manuscript. Additionally, we thank Roger Marsh for his programming assistance, and Angela Colcombe and Trina Ragain for their assistance in collecting data. Correspondence should be addressed to A. F. Kramer, Beckman Institute, University of Illinois, 405 North Mathews Ave., Urbana, IL 61801 (e-mail: akramer@ s.psych.uiuc.edu). terest relative to events depicted in the scene. The researchers employed a flicker paradigm, in which a gray field is presented for $80 \mathrm{msec}$ between successive views of the original and changed scenes ( $240 \mathrm{msec}$ each). These images alternate until the observer responds or until $60 \mathrm{sec}$ have elapsed. The results showed that observers had difficulty detecting change (averaging $7.8 \mathrm{sec}$ to detect change), suggesting that the flickering blank fields masked the transients occurring with the change onset. Rensink et al. found that changes to items of central interest were detected faster than changes to items of marginal interest, even though marginal interest changes tended to be larger on average ( $22 \mathrm{deg}^{2}$ of visual angle compared with $18 \mathrm{deg}^{2}$ of visual angle for central interest changes). Rensink et al. proposed that objects of central interest probably attracted attention via higher level cognitive processes.

In the present study, we examined the relationship between perceptual change detection and attention by relating individual differences in attentional breadth to observers' ability to detect changes in driving scenes. More specifically, we derived measures of the functional field of view (FFOV) for each of the participants and then examined the relationship between these measures and the speed with which they could detect a variety of different types of changes in driving scenes.

The FFOV represents the spatial area that is needed to successfully perform a specific visual task without invoking eye or head movements (Ball, Roenker, \& Bruni, 1990; Mackworth, 1965, 1976). Typically, the FFOV is defined as the distance from fixation at which a given task is reliably performed. FFOV tasks generally consist of detecting, identifying, or localizing targets in the periphery, 
and increasing task demands generally result in a decrease in the size of the FFOV.

The FFOV is a useful tool for revealing individual differences in attentional breadth. One example of this is that the size of the FFOV declines with age (e.g., Ball, Beard, Roenker, Miller, \& Griggs, 1988; Scialfa, Thomas, \& Joffe, 1994; Seiple, Szlyk, Yang, \& Holopigian, 1996; Sekuler \& Ball, 1986). Ball et al. (1988) found that agerelated differences in the FFOV were magnified when targets were presented with distractors. Furthermore, a reduction in an older driver's FFOV predicts degraded driving performance (Isler, Parsonson, \& Hansson, 1997; Rizzo, Reinach, McGehee, \& Dawson, 1997; Sims, Owsley, Allman, Ball, \& Smoot, 1998), a skill that would seem to rely on an ability to detect change in the environment.

Given these findings, we had three purposes in conducting the present research. First, we examined the hypothesis that there would be a negative correlation between change detection latency and attentional breadth (FFOV). This hypothesis is based on the assumption that changes in scenes are detected by sequentially sampling portions of the scenes with an attentional window or aperture (à la Rensink et al., 1997), and therefore observers with broader attentional windows should be able to detect changes with fewer samples. Second, given previous findings of reduced FFOVs with increasing age, we hypothesized that perceptual change performance would decline with age. Including both young and old adults in the study also served to increase individual differences in FFOV or attentional breadth, thereby increasing the power to detect a relationship between perceptual change detection and FFOV. Finally, we examined the influence of several factors that have been shown to influence attentional control (i.e., salience, meaning, and eccentricity of changes) on perceptual change detection in complex scenes.

\section{METHOD}

\section{Participants}

A total of 51 people participated in the study. The 25 younger participants (13 women, 12 men) were recruited from the University of Illinois and ranged in age from 18 to 33 years $(M=23$ years). The 26 older participants ( 18 women, 8 men) were recruited from the local community and ranged in age from 55 to 80 years $(M=$ 68 years). Each participant had corrected visual acuity better than $20 / 40$, had possessed a valid driver's license for the previous 2 years, and drove over 25 miles per month. Education information was not available for all participants, but analyses indicated that the mean number of years of education for younger adults (16.1 years, $S D=3.1, n=17$ ) was not statistically different from the mean education for older adults [14.5 years, $S D=2.4, n=24 ; t(39)=1.49$, $p<.07]$. Participants were compensated at a rate of $\$ 6$ per hour for their participation in the experiment.

\section{Apparatus}

A Micron Millenia MME computer with a $12 \times 16$ in. Viewsonic monitor was employed. Participants rested their chins on a chinrest $56 \mathrm{~cm}$ from the screen. A Fresnel lens was used to eliminate the accommodation cues and to effectively present the stimuli at a distance approaching optical infinity. The Fresnel lens also increased the subjective size of the image region.

\section{Perceptual Change Task}

The perceptual change task was conducted in the same fashion as that of Rensink et al. (1997). Each trial consisted of an original image (A) and a modified version $\left(\mathrm{A}^{\prime}\right)$, which were displayed in the sequence $\mathrm{A}, \mathrm{A}, \mathrm{A}^{\prime}, \mathrm{A}^{\prime}$. Gray blank fields were placed between successive images to eliminate apparent motion across image displays. Each image was displayed for $240 \mathrm{msec}$ and each blank screen (gray field) for $80 \mathrm{msec}$.

Eighty digital photographs of scenes taken from a driver's perspective inside a car were manipulated in the experiment (80 experimental trials). Images were presented to the observers as approximately $25 \mathrm{deg}$ wide and $20 \mathrm{deg}$ high. The modified version of each scene involved a change in a single object's color, location, or presence. Objects were recognizable items such as buildings, cars, or roadways. Changes could entail such things as a building disappearing, a green car changing into a red car, or a pedestrian moving closer to the roadway. The objects and their changes were categorized along three dimensions: eccentricity, meaningfulness, and salience. Eccentricity was measured according to the changed object's distance (in degrees of visual angle) from the center of the image, which coincided with the observer's initial fixation point prior to the start of a trial. Central changes fell within 6 deg visual angle from the center of the image, while peripheral changes fell outside a radius of $6 \mathrm{deg}$ from center. Measures of object and change meaningfulness and salience were determined separately in pilot studies (discussed below).

Participants were instructed that they would be viewing scenes taken from the driver's perspective. They were told to fixate the center of the screen and to indicate to the experimenter when they were ready. Once the experimenter began a trial, observers were allowed to search freely for the image change. When they detected the change, observers were instructed to press the mouse button and then to verbally describe the change. Although they were allowed to view the alternating scenes up to $1 \mathrm{~min}$, participants were instructed to respond as quickly and as accurately as possible.

Participants were told of the types of changes possible (i.e., object's color, location, or presence) prior to beginning the experiment and were given two practice trials to familiarize them with the task. Images and image changes (i.e., meaningfulness, salience, and eccentricity) were presented in a random order for each participant. The dependent variables were the response time (RT) needed to detect the change and the accuracy of the detection.

Four factors were evaluated in the perceptual change task (i.e., age, meaningfulness, salience, and eccentricity). Age served as a between-subjec ts factor, and the remaining three factors were within subjects and were randomized within trial blocks.

The meaningfulness and salience characteristics of both the object and its change are likely to have a high degree of overlap, as in the case of a disappearing semi-truck (i.e., a salient, meaningful vehicle undergoing a very salient, meaningful change), but the correspondence is not perfect. For example, changing the color of a salient, meaningful object (e.g., the semi-truck) to a slightly lighter shade of gray would be a hardly noticeable, nonmeaningful change. Therefore, the meaningfulness and salience characteristics of both the object and its change were determined in two separate pilot studies. The first study examined the characteristics of the change (i.e., a modification of the properties of an object varying over time and occasionally over spatial location), while the second study examined characteristics of the object undergoing change (i.e., a recognizable item with consistent spatiotemporal properties).

The first pilot study consisted of 14 younger ( $M=22$ years) and 10 older adults ( $M=72$ years). Participants saw two images of a scene (original and modified) on color printed pages in a notebook. Once they had correctly identified the change between the two im- 
ages, they were asked to rate the change according to a 6-point Likert scale. They rated the 82 changes (including the two practice pictures) on one dimension (i.e., meaningfulness or salience) before rating them on the other dimension. Order was counterbalanced across participants.

Meaningfulness was defined to the raters as the relevance or importance of the change to driving performance. For example, changing the color of a restaurant sign should be given a low meaningfulness rating, while changing the color of a stoplight should be rated high. Salience was defined to the raters in terms of low-level perceptual factors. For example, a large, bright, noticeable change should be rated as highly salient, while a small, dim, difficult-to-see change should be rated low.

The second pilot study was conducted in the same fashion as the first with the following exception: Participants ( 6 young adults, $M=$ 22 years; 6 older adults, $M=76$ years) were asked to rate a single object in each of the 82 scenes according to the degree of the object's perceived meaningfulness and salience. The participants were not aware of object modifications, since they only viewed a single image of each scene.

Meaningfulness was again defined in terms of the object's relevance or importance to driving behavior (e.g., a stop sign has high object meaningfulness, a building has low object meaningfulness). Salience referred to the object's prominence or visibility (e.g., a large building has high object salience, a license plate has low object salience).

Analyses of subjective ratings to the 80 driving scenes (excluding the two scenes used in practice trials) were conducted to examine the range of variability in ratings of the pictures on the meaningfulness and salience scales and to determine the degree of similarity of meaningfulness and salience ratings for older and younger adults. Given the high correlation between meaningfulness and salience ratings for the objects and changes $(r=.50, .77$, respectively), results will be reported in terms of the meaningfulness and salience ratings of the change. It should also be noted that we conducted a complete set of analyses on the basis of the object ratings, and these results are consistent with the results that follow (which are based on the ratings of the change).

The mean and standard deviation of the median ratings for salience were 2.95 and 1.29 , respectively. The comparable ratings for meaningfulness were 2.59 and 1.98 , respectively. Thus, raters judged the scene changes as varying to a greater extent in meaningfulness than in salience. No significant differences were found between young and old observers for mean ratings of meaningfulness $[t(21)=-.78, p<.44]$; however, older observers rated changes as more salient than younger observers $[t(21)=-3.43$, $p<.003]$. Cronbach's alpha reflected high degrees of consistency for each of the rated dimensions (.91 and .94 for the rated meaningfulness and salience of the change, respectively).

For the purpose of analysis of the perceptual change performance, the 80 driving scenes were divided into four categories (i.e., low meaning/low salience, low meaning/high salience, high meaning/ low salience, high meaning/high salience) on the basis of the median ratings across raters. We next examined whether the range of differences between low and high categories was equivalent for the meaningfulness and salience factors. We evaluated the relative differences between low and high salience and meaningfulness ratings of the pictures in terms of standard deviation units. For example, averaging across all pictures in the low meaningfulness/low salience category produced mean meaningfulness and salience scores of 0.4 and 1.4, respectively. The comparable mean ratings for meaningfulness and salience of pictures in the high meaningfulness/low salience category were 4.1 and 1.75 , respectively. The standard deviations for the meaningfulness and salience ratings were 1.98 and 1.29 , respectively. Thus, the difference between high and low meaningfulness for low-salience pictures (high-low meaningfulness for all low-salience pictures) was $(4.1-0.4) / 1.98$, or $1.86 S D$ units. For the remaining categories, the calculated values were $1.87,1.63$, and 1.67 SD units for high-low meaningfulness for high-salience pictures, and high-low salience for low- and high-meaningfulness pictures, respectively. Because the difference between high and low in standard deviation units was approximately the same (i.e., $1.63-$ 1.87) across categories, this analysis suggests that the meaningful changes in our pictures and the salience changes in our pictures were equated reasonably well.

\section{Attentional Breadth Task}

A measure of each observer's attentional breadth (FFOV) was obtained using a visual search task in which observers searched for an oblique target (tilted $20 \mathrm{deg}$ left of vertical) appearing among 11 vertical distractors.

Targets and distractors each subtended 2 deg of visual angle and could appear randomly at one of three eccentricities (10,20, and 30 deg from fixation) along eight radial meridians for a total of 24 possible positions. At least one, but no more than two, distractors occupied each of the eight meridians during a trial.

Observers were instructed to click the mouse button after fixating the center fixation cross in order to begin a trial. The stimuli subsequently appeared for $250 \mathrm{msec}$, followed by a response display, which remained present until a response was made. Observers then moved the mouse to one of the 24 possible target positions to indicate their response. Instructions emphasized accuracy of responding. Observers completed two blocks of 144 trials each, with 24 practice trials before each block.

Accuracy in localizing targets as a function of target eccentricity determined the size of the FFOV. Specifically, the size of the FFOV was estimated as the eccentricity at which localization accuracy was $50 \%$ (chance performance was approximately $4 \%$ since observers could localize a target at 1 of 24 locations on the computer monitor). In order to eliminate the hypothesis that age differences at eccentric locations could be due to visual problems, two additional blocks were completed with oblique targets appearing without distractors. This baseline condition ensured that the older participants could indeed detect peripheral stimuli. In fact, older adults achieved greater than $95 \%$ accuracy at all three target eccentricities on the baseline task. Since the FFOV task has been extensively studied, we refer the interested reader to Ball et al. (1988), Scialfa et al. (1994), and Seiple et al. (1996) for a more complete treatment of this topic.

\section{RESULTS}

Two sets of analyses were performed on the data. We first report an analysis of the influence of age, salience, meaningfulness, and eccentricity of the change on perceptual change detection performance. ${ }^{1}$ Second, we examined the relationship between performance on the attentional breadth (FFOV) task and performance on the perceptual change task.

\section{Change Detection Performance}

Only correct trials were used in the RT analysis. Additionally, RTs greater than $3 S D$ from the mean for each age group were discarded prior to calculating mean RTs. One younger adult did not complete the experiment, and the data were not included in the analyses.

The RT data were positively skewed (skewness = 1.97); thus a logarithmic transformation was applied. This served to improve normality (skewness $=0.21$ ) and to re- 

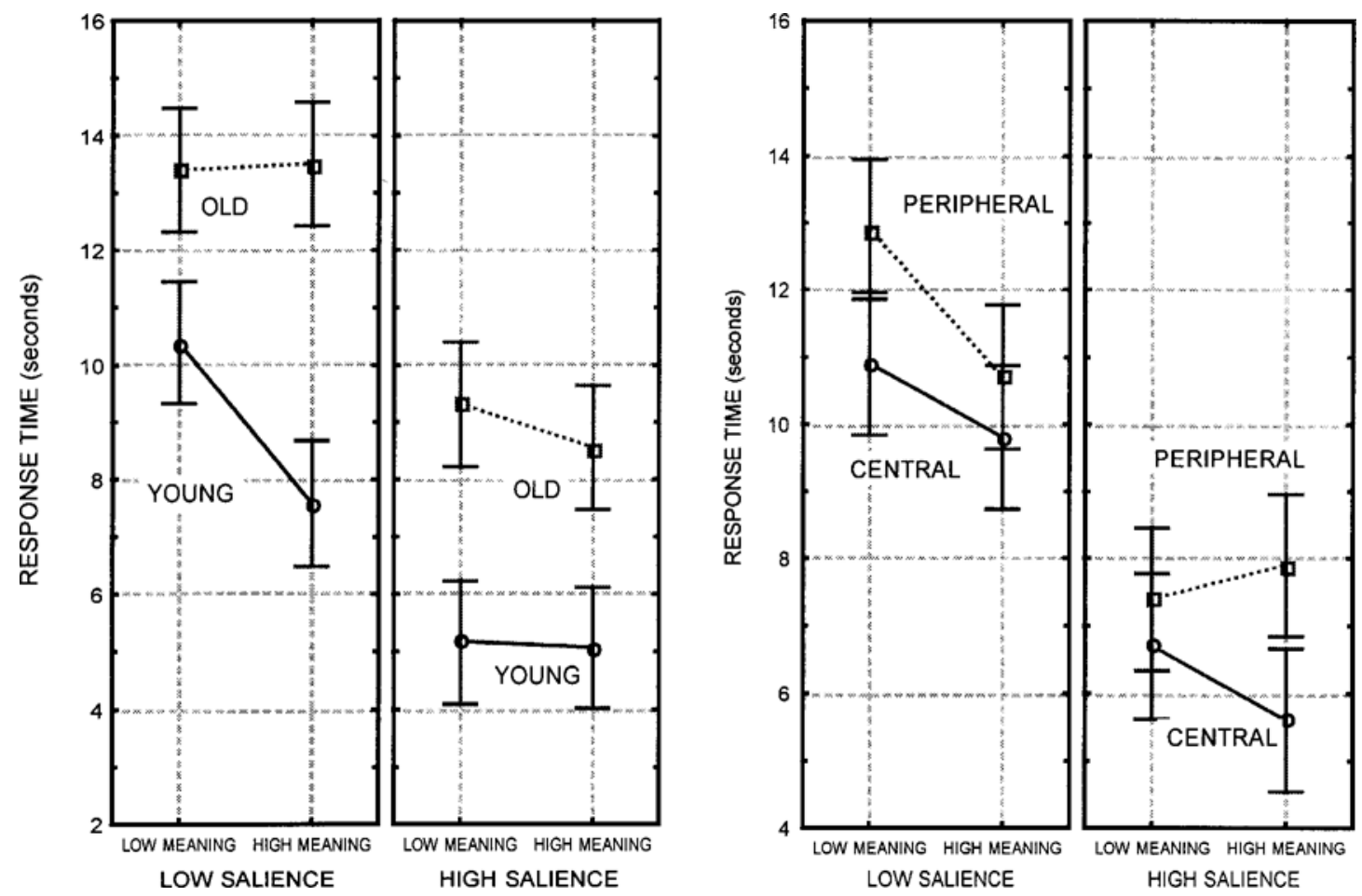

Figure 1. Left panel: Mean RTs for the perceptual change task for the age $\times$ meaningfulness $\times$ salience interaction. Right panel: Mean RTs for the perceptual change task for the eccentricity $\times$ meaningfulness $\times$ salience interaction.

duce the influence of outliers (especially in the older age group). The logarithmic transformed perceptual change detection RTs were submitted to a four-way mixed mode analysis of variance (ANOVA) with age as a betweensubjects factor and meaningfulness (high and low), salience (high and low), and eccentricity (central and peripheral) as within-subjects factors. ${ }^{2}$

Main effects were significant for all four factors (age, eccentricity, meaningfulness, and salience). Younger adults performed significantly faster than older adults [6.8 and $10.9 \mathrm{sec}$, respectively; $F(1,48)=41.02, p<.001$ ]; central changes were detected more quickly than peripheral ones [7.9 and $9.4 \mathrm{sec}$, respectively; $F(1,48)=35.14$, $p<.001]$; and change detection was enhanced for meaningful changes $[$ low $=9.0$, high $=8.2 \mathrm{sec} ; F(1,48)=9.65$, $p<.003]$ as well as for salient changes [low $=10.9$, high $=6.8 \mathrm{sec} ; F(1,48)=313.93, p<.001]$.

A significant two-way interaction was obtained between age and salience $[F(1,48)=6.53, p<.014]$, and a marginally significant two-way interaction was obtained between age and meaningfulness $[F(1,48)=3.85, p<$ .056]. The age $\times$ eccentricity interaction was not significant, although there are several potential explanations. One reason is that we did not control for eye movements (e.g., Scialfa et al., 1994). Furthermore, the age $\times$ eccentricity interaction is not consistently found in the literature (e.g., Foster, Behrmann, \& Stuss, 1995; Plude \& Doussard-Roosevelt, 1989).

These interactions were mediated by the three-way interaction between age, meaningfulness, and salience $[F(1,48)=7.94, p<.007]$. This interaction is illustrated in Figure 1 (left panel). Scheffé post hoc analyses indicated that increasing meaningfulness had no effect on performance for either age group when changes were highly salient. On the other hand, when salience of the change was low, increasing meaningfulness aided the performance of young $(p<.001)$, but not old adults.

A significant three-way interaction was also found for eccentricity $\times$ meaningfulness $\times$ salience $[F(1,48)=$ $9.64, p<.003]$. This interaction is illustrated in the right panel of Figure 1. Post hoc analyses revealed that central changes were detected faster than peripheral changes only when changes were of both high meaning and high salience $(p<.001)$ and that varying meaningfulness did not influence performance when changes were both peripheral and of low salience $(p<.07)$.

Mean accuracy ratings for younger and older groups for each level of eccentricity, meaningfulness, and salience are provided in Table 1. Inaccurately identifying the photograph change and not finding the change within the 1min time limit were both considered errors. A four-way mixed mode ANOVA was performed on the accuracy 
Table 1

Mean Accuracy for Younger and Older Groups by Level of Eccentricity, Meaningfulness, and Salience

\begin{tabular}{lrrrrr}
\hline & \multicolumn{2}{c}{ Younger } & & \multicolumn{2}{c}{ Older } \\
\cline { 5 - 6 } \multicolumn{1}{c}{ Condition } & $M$ & $S D$ & & $M$ & $S D$ \\
\hline Central low meaning low salience & 93.7 & 9.6 & & 77.2 & 16.3 \\
Central low meaning high salience & 99.0 & 5.1 & & 94.9 & 10.8 \\
Central high meaning low salience & 96.7 & 7.6 & & 79.6 & 22.4 \\
Central high meaning high salience & 99.7 & 1.4 & & 92.2 & 8.3 \\
Peripheral low meaning low salience & 90.2 & 8.0 & & 71.9 & 19.7 \\
Peripheral low meaning high salience & 99.3 & 2.4 & & 90.0 & 7.8 \\
Peripheral high meaning low salience & 91.7 & 10.2 & & 71.3 & 19.2 \\
Peripheral high meaning high salience & 92.6 & 5.1 & 86.0 & 8.9 \\
\hline
\end{tabular}

Note-The values represent mean percentages of correctly identified changes for younger $(n=24)$ and older $(n=26)$ adults.

data, with age as a between-subjects factor and meaningfulness, salience, and eccentricity as within-subjects factors. Main effects were significant for age $[F(1,38)=$ $39.8, p<.001]$, eccentricity $[F(1,38)=31.7, p<.001]$, and salience $[F(1,48)=64.0, p<.001]$. Younger adults were more accurate overall in detecting changes than were older adults. Additionally, accuracy was higher for changes occurring centrally compared with peripheral changes, and changes with high salience compared with those with low salience. Significant two-way interactions were found for age $\times$ salience $[F(1,48)=19.3, p<.001]$ and meaningfulness $\times$ salience $[F(1,48)=4.7, p<.03]$. Older adults were hurt more by low levels of salience than were younger adults, perhaps due to a ceiling effect for younger adults' accuracy. There was a larger difference between low and high salience at low levels of meaningfulness relative to high levels of meaningfulness.

\section{Relationship Between FFOV \\ and Change Detection Performance}

The second set of analyses examined the relationship between performance on the FFOV task and performance on the perceptual change task. Recall that the size of the FFOV was determined on the basis of observers' accuracy in localizing oblique targets appearing among 11 vertical distractors at various eccentricities (i.e., the $50 \%$ accuracy point on a linear function relating localization accuracy to eccentricity).

The scatterplot representing the relationship between the estimated size of the FFOV and perceptual change detection latency for all participants is depicted in Figure 2. A larger FFOV corresponded to faster detection of object changes $(r=-.68, p<.001)$. This trend was found for both younger and older adults when analyzed separately $(r=-.50, p<.01 ; r=-.51, p<.01$, for younger and older adults, respectively).

To examine this issue further, a correlation was calculated between FFOV size and detection latency for central and peripheral changes. For centrally located changes, the correlation was $-.54(p<.01)$. For scene changes occurring in the periphery, the correlation was $-.66(p<$ $.01)$. Thus, the size of the FFOV does appear to be re- lated to change detection latency for central changes and perhaps even more strongly for peripheral changes.

\section{DISCUSSION}

The goals of the present research were to examine the effects of age and characteristics of the change on change detection performance and to determine the relationship between breadth of attention (FFOV) and perceptual change detection. Overall, the results support three conclusions: (1) Change is difficult to detect under flicker conditions, especially for older adults; (2) detection is mediated by the characteristics of the change; and (3) attention is related to change detection.

\section{Age, Change Characteristics, and Change Detection}

The results were generally consistent with the perceptual change blind ness literature, with the average time to detect a change under the best of circumstances approximately $5 \mathrm{sec}$. Furthermore, we found that the characteristics of the change (eccentricity, salience, and meaningfulness) had interacting effects on change detection performance.

Our results shed light on the role of top-down and bottom-up factors in change detection. Meaningfulness had a smaller impact on performance than did salience, especially for the older adults. This occurred despite the fact that difference between high and low meaningfulness of changes to pictures was judged to be larger $(M=$ $1.87 S D$ units) than the difference between high- and low-salience changes ( $M=1.65 S D$ units) by our raters. These results suggest that attention guided by meaningfulness (i.e., higher level processes) is not as powerful as attention guided by salience (i.e., lower level visual processes) in change detection, especially for older adults. One possible explanation for the relatively modest effect of meaningfulness is that the context might not have been sufficiently realistic for participants to consider themselves "in the driver's seat." We plan to investigate this in future research.

Salience, through low-level perceptual means, appears to be quite effective in drawing attention to a change. Although salience has not been directly assessed in previous change detection experiments, the results obtained here are consistent with visual search results. Nothdurft (1993) found that local differences in salience (due to color, motion, luminance, or orientation) provided similarly fast detection of the target, which, if nonsalient, could be detected only by serial processing. This might also hold true in change detection, in such a way that salient changes to objects (along these dimensions) are quickly detected, but nonsalient changes are detected by slow, serial processing.

Rensink et al. (1997) found some evidence for change characteristics influencing perceptual change detection. Specifically, they found that changes to items of central interest were detected faster than changes to items of 


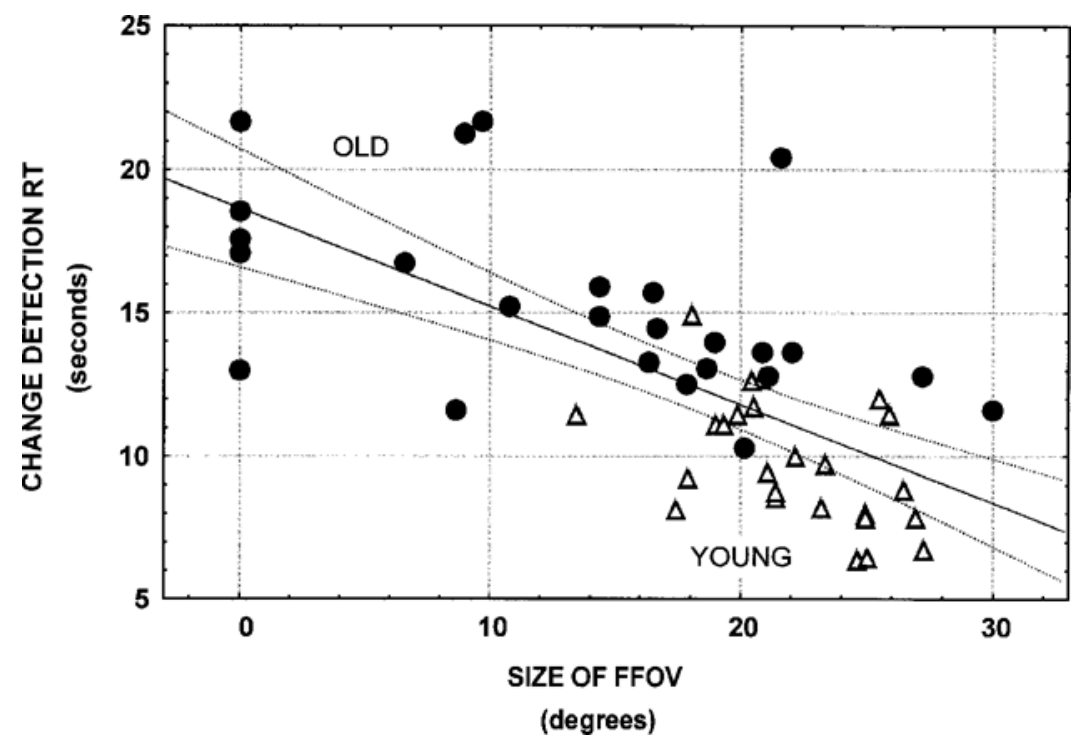

Figure 2. Scatterplot for the FFOV (attentional breadth) measure and perceptual change detection RT. The unfilled triangles represent data points for the younger observers; the filled circles represent older observers. The solid line represents the bestfitting linear function relating change detection RT to FFOV size. The broken lines represent the $95 \%$ confidence limits.

marginal interest, even though marginal interest changes tended to be larger on average ( $22 \mathrm{deg}^{2}$ of visual angle compared with $18 \mathrm{deg}^{2}$ of visual angle for central interest changes). Although these results may, at first glance, appear to suggest a different conclusion than that reached in the present study (i.e., that interest [meaningfulness] might be more relevant than size [salience]), there are two issues that need to be considered. First, the size of the change was not the only criterion for highly salient changes in our experiment. Salience also encompassed changes in luminance and color. Second, items of "central interest" using Rensink et al.'s terms do not exactly correspond to meaningfulness as we defined it. For example, if the car in the center of a picture changed color, this would probably be of central interest in Rensink's terms, but of little "meaning" to the task of driving.

Finally, our finding that older adults had more difficulty detecting change under most circumstances adds a new dimension to the present literature on change detection. It is interesting to note that older and younger adults show differences in detecting changes (i.e., that meaningfulness had very little influence on older adult change detection performance compared with that of younger adults), but not altogether unsurprising given other findings for age-related differences on various visual search tasks (e.g., Gilmore, Tobias, \& Royer, 1985; Humphrey \& Kramer, 1997; Rogers \& Fisk, 1991). The abundance of findings for qualitative (as well as quantitative) differences for older adults on a variety of visual search tasks implies that visual search for change in a simulated saccade paradigm may exhibit age-related differences as well.

\section{Attentional Breadth and Change Detection}

We found a strong correlation between breadth of attention and change detection, such that a smaller FFOV corresponded to slower change detection. The correlation was slightly stronger between FFOV size and detection of peripheral changes. This finding strengthens the claim for a relationship between efficiency of change detection and attention (Rensink et al., 1997). Furthermore, our results suggest that a particular aspect of attentionthe breadth of attention - plays an important role in perceptual change detection, presumably by reducing the number of attentional samples required to detect a change. However, this finding does not preclude the role of other factors in performance, such as working memory or perceptual speed, and does not rule out the possibility that change may be detected without requiring attention (Fernandez-Duque \& Thornton, 2000; Rensink, 1998). We are currently investigating these issues.

In summary, it appears that visual attention plays an important role in detecting change since individual differences in breadth of attention reliably correlated with perceptual change detection. Furthermore, salient scene characteristics were more responsible for driving attention to change than meaningful change characteristics, especially for older adults. Although we are not perfect perceivers of the environment, the breadth of our attention 
affects our ability to detect change and allows us to see (or not to see) the big picture.

\section{REFERENCES}

Ball, K. B., Beard, B. L., Roenker, D. L., Miller, R. L., \& Griggs, D. S. (1988). Age and visual search: Expanding the useful field of view. Journal of the Optical Society of America, A, 5, 2210-2219.

Ball, K. B., Roenker, D. L., \& Bruni, J. R. (1990). Developmental changes in attention and visual search throughout adulthood. In J. Enns (Ed.), The development of attention: Research and theory. Advances in psychology (Vol. 69, pp. 489-508). Amsterdam: NorthHolland.

Fernandez-Duque, D., \& Thornton, I. M. (2000). Change detection with awareness: Do explicit reports underestimate the representation of change in the visual system? Visual Cognition, 7, 323-344.

Foster, J. K., Behrmann, M., \& Stuss, D. T. (1995). Aging and visual search: Generalized cognitive slowing or selective deficit in attention? Aging \& Cognition, 2, 279-299.

Gilmore, G. C., Tobias, T. R., \& Royer, F. L. (1985). Aging and similarity grouping in visual search. Journal of Gerontology, 40, 586592.

Grimes, J. (1996). On the failure to detect changes in scenes across saccades. In K. Akins (Ed.), Perception (Vol. 5, pp. 89-110). New York: Oxford University Press.

Henderson, J. (1997). Transsaccadic memory and integration during real-world object perception. Psychological Science, 8, 51-55.

Humphrey, D. G., \& Kramer, A. F. (1997). Age differences in visual search for feature, conjunction, and triple-conjunction targets. Psychology \& Aging, 12, 704-717.

IRWIN, D. (1996). Integrating information across saccadic eye movements. Psychological Science, 5, 94-100.

Isler, R. B., Parsonson, B. S., \& Hansson, G. J. (1997). Age related effects of restricted head movements on the useful field of view of drivers. Accident Analysis \& Prevention, 29, 793-801.

Mackworth, N. H. (1965). Visual noise causes tunnel vision. Psychonomic Science, 3, 67-68.

Mackworth, N. H. (1976). Stimulus density limits the useful field of view. In R. A. Monty \& J. Senders (Eds.), Eye movements and psychological processes (pp. 307-321). Hillsdale, NJ: Erlbaum.

McConkie, G. W., \& Currie, C. B. (1996). Visual stability across saccades while viewing complex pictures. Journal of Experimental Psychology: Human Perception \& Performance, 22, 563-581.

NothdURF, H. (1993). Saliency effects across dimensions in visual search. Vision Research, 33, 839-844.

Plude, D. J., \& Doussard-Roosevelt, J. A. (1989). Aging, selective attention, and feature integration. Psychology \& Aging, 4, 98-105.

Rensink, R. A. (1998). Mindsight: Visual sensing without seeing. Investigative Ophthalmology \& Visual Science, 39, S631.
Rensink, R. A., O'Regan, J. K., \& Clark, J. J. (1997). To see or not to see: The need for attention to perceive changes in scenes. Psychological Science, 8, 368-373.

Rizzo, M., Reinach, S., McGehee, D., \& Dawson, J. (1997). Simulated car crash and crash predictors in drivers with Alzheimer's disease. Archives of Neurology, 54, 545-551.

Rogers, W. A., \& FISK, A. D. (1991). Are age differences in consistentmapping visual search due to feature learning or attention training? Psychology \& Aging, 6, 542-550.

Scialfa, C. T., Thomas, D. M., \& Joffe, K. M. (1994). Age differences in the useful field of view: An eye movement analysis. Optometry \& Vision Science, 71, 736-742.

Seiple, W., Szly K, J. P., YAng, S., \& Holopigian, K. (1996). Age-related functional field losses are not eccentricity dependent. Vision Research, 36, 1859-1866.

SeKuler, R., \& Ball, K. B. (1986). Visual localization: Age and practice. Journal of the Optical Society of America A, 3, 864-867.

Sims, R. V., Owsley, C., Allman, R. M., Ball, K., \& Smoot, T. M. (1998). A preliminary assessment of the medical and functional factors associated with vehicle crashes by older adults. Journal of the American Geriatrics Society, 46, 556-561.

\section{NOTES}

1. Differences across change types were not equated. Nonetheless, within each change type category (i.e., color, location, or presence), trends supported the above findings. Specifically, the 13 changes in location were the easiest to detect overall, averaging $9.26 \mathrm{sec}$. Presence changes (i.e., add/delete) were more frequent (i.e., 47 changes), but took longer to detect $(12.13 \mathrm{sec})$. Finally, the 20 color substitutions took the longest to detect at $13.96 \mathrm{sec}$. It is possible that location changes were easier to identify because observers had two opportunities (i.e., locations) to detect the change, whereas presence and color changes occurred in a single spatial location.

Further analyses revealed that young observers were faster than the old in each type of change (a benefit of 5.4, 5.1, and $3.1 \mathrm{sec}$ for location, presence, and color changes, respectively). The benefit of high meaningfulness was largest for location changes $(5 \mathrm{sec})$, but was practically absent for presence and color changes $(.5 \mathrm{sec})$. Likewise, the benefit of salience was largest for location changes $(8.4 \mathrm{sec})$, followed by presence changes $(4.9 \mathrm{sec})$ and color changes $(2.6 \mathrm{sec})$.

2 . Analyses conducted on the raw RT data produced similar, although weaker, effects due to the influence of outliers. Of particular interest are the three-way interactions. The age $\times$ meaning $\times$ salience interaction was marginally significant $[F(1,48)=3.85, p<.0554]$, while the eccentricity $\times$ meaning $\times$ salience interaction was not significant $[F(1,48)=$ $0.36, p<.55]$.

(Manuscript received March 9, 1999; revision accepted for publication January 27, 2000.) 